\title{
- Integrating Relational Psychoanalysis and EMDR: Embodied Experience and Clinical Practice
}

\author{
Hemda Arad. New York, NY: Routledge, 2018, 206 pages, \$44.95 (paperback)
}

Hemda Arad's book is a poetic introduction to eye movement desensitization and reprocessing (EMDR) for practitioners of relational psychoanalysis. It contains a brief description of EMDR, including the adaptive information processing (AIP), Natalie Robinson's 2016 eight-stage flow chart, and many references to "big T and little $t$ traumas." It has a good description of the history and development of relational psychoanalysis, Bowlby's attachment styles, and much of the research and physiology of attachment and trauma. And it speaks about many cases and how EMDR helped analytic clients move forward in their process.

I loved the chance to learn more about relational psychoanalysis and to read some of Arad's soaring prose about the process. Here are her words about the musicality of bilateral stimulation (BLS) during processing:

In addition, the EMDR protocol, in particular during BLS sets, sends the patient into an internal dialogue that resembles early or significant communication with a caregiver. As a consequence, the patient engages with negative representations, sourced from the small $t$ trauma of a daily misattunement every child experiences, or the big $T$ trauma of gross violations of the mind and body by a person in the life of the child. Both repetitive imagery and the reconstructed imagery in the EMDR protocol evoke a mini-idiosyncratic world determined by their own rhythmic cadence. It is within these all-encompassing affective dances that the possibility of distinguishing between a dance that seems to be the only tune possible, and other variations of the musical building blocks that can potentially spell a new, more adaptive tune of the self in relation to the other and its environment can emerge. (pp. 98-99)

While I was loving the language and agreeing with Arad's conclusions about attachment, trauma, transference, research, and the healing process, I found myself frustrated by what was missing. When she spoke about using EMDR with clients, she rarely showed the dialogue, her methods of introducing EMDR into the sessions, and the step-by-step working through that could show her readers exactly how it happened. She would go into lovely descriptions of transformation and even theories about why it worked, while I kept wanting more how. I could see that she knew what she was doing, and I wanted to know what the exact steps were that she took to get her clients to the new plateaus. Sometimes, I couldn't tell if she was using BLS inside or outside the Standard Protocol. Sometimes it was clearly explained.

And sometimes the lovely prose was too much for me. I found myself muttering more than once, "Hemda, cut to the chase!"

I think this is a good book for relational psychoanalysts, whether or not they do EMDR, and for EMDR practitioners who are interested in psychoanalysis. I think it would be extremely frustrating for people who don't have a foot in either camp or who are looking for a how-to manual of integrating these modalities.

And I need to disclose that I know Hemda Arad, I've consulted to her. She's a skilled therapist. And I like her. 